\title{
A LOCALIZAÇÃO DAS PUBLICAÇÕES E A IDENTIFICAÇÃO DE TÓPICOS QUENTES EM SUSTENTABILIDADE: UMA PRIMEIRA APROXIMAÇÃO USANDO O WEB OF SCIENCE
}

\author{
Lúcia Rejane da Rosa Gama Madruga \\ Doutora em Agronegócios pela Universidade Federal do Rio Grande do Sul - UFRGS \\ Professora da Universidade Federal de Santa Maria - UFSM \\ lucia.rejane@hotmail.com
}

\section{RESUMO}

Tendo como preocupação central o estudo da sustentabilidade, este artigo analisa as publicações sobre este tema no banco de dados Web of Science, procurando a localização espaço-temporal destas publicações, assim como a identificação de temas de fronteira (tópicos quentes) nesta área do conhecimento. Esse estudo revelou, entre outras questões, que cresce o volume de publicações nos anos mais recentes, concentrando-se nos Estados Unidos, tendo principal fonte o Journal Ecological Economics e como principal assunto as ciências ambientais. Os temas quentes são relacionados às disciplinas de gestão e de desenvolvimento, à dimensão ambiental e à água e ao solo, elementos do ecossistema.

Palavras-chaves: Publicações; Sustentabilidade; Tópicos quentes.

\section{THE LOCATION OF THE PUBLICATIONS AND THE IDENTIFICATION OF HOT TOPICS IN SUSTAINABILITY: A FIRST APPROXIMATION USING THE WEB OF SCIENCE}

\begin{abstract}
This article analyzes publications in the database Web of Science, on the central concern of sustainability, looking for the space-time location of these publications as well as the identification of frontier topics (hot topics) within this area of knowledge. The study revealed an increasing volume of publications in recent years concentrated in the United States with the main source 'Journal Ecological Economics' and the main subject being the environmental sciences. The hot topics are related to the disciplines of management and development, environmental and water and soil elements of the ecosystem.
\end{abstract}

Keywords: Sustainability; Hot topics; Publication. 


\section{INTRODUÇÃO}

A preocupação com o futuro do planeta terra e com o reflexo do modelo econômico vigente sobre os estoques de capital natural tem levado à proposição de um novo modelo de desenvolvimento alicerçado na perspectiva da sustentabilidade, que prevê a transição para um sistema industrial pautado em uma mentalidade e em uma escala de valores muito diferentes dos valores dominantes na sociedade atual. Neste novo modelo, à dimensão econômica se somam as dimensões social e ambiental e se processam reflexões sobre o paradigma de bem-estar vigente, procurando-se alternativas sustentáveis no futuro, ou seja, que atendam as necessidades da sociedade atual e, ao mesmo tempo, tratem com equidade as gerações futuras, no que diz respeito às questões ambientais e à qualidade de vida.

Assim como crescem as preocupações com o contexto da sustentabilidade aumentam as publicações sobre este tema, tornando o trabalho de estudantes e pesquisadores iniciantes bastante árduo, no que tange à busca de questões de pesquisa relevantes que sejam do interesse da comunidade científica neste campo do conhecimento. Ao dar início a uma tese ou pesquisa, Banks (2006) sugere que é pertinente se questionar sobre: quanto já foi feito neste tópico? Este é um "hot tópic"? Quantas pessoas se interessem pelo resultado da minha tese ou pesquisa? O autor reforça que a riqueza de informação de hoje dificulta a resposta a tais perguntas sem uma grande procura inicial na literatura.

Inserido neste contexto, este artigo se propõe a responder a seguinte questão de pesquisa: qual a localização espaço-temporal das publicações sobre sustentabilidade, disponibilizadas no banco de dados Web of Scienc, e quais os temas considerados "quentes" nesta área do conhecimento?

Este artigo está estruturado em cinco seções, incluindo as notas introdutórias. A segunda seção apresenta uma revisão sobre o tema sustentabilidade onde se buscou identificar as principais dimensões conceituais, bem como os tópicos que mais fortemente se relacionam a este conceito na literatura. $\mathrm{Na}$ terceira seção é apresentado o método. $\mathrm{Na}$ quarta, os resultados. Na quinta, as considerações finais e, por fim, as referências.

\section{SUSTENTABILIDADE: PRESSUPOSTOS INICIAIS DE UM CONCEITO EM CONSTRUÇÃO}

A partir da definição de desenvolvimento sustentável, formulada com o consenso de comunidades internacionais, a preocupação com o futuro do planeta terra e com a qualidade de vida das gerações futuras incorporou as inquietações de vários agentes sociais em busca de soluções compartilhadas para os problemas decorrentes do uso inadequado do capital natural.

A definição de desenvolvimento sustentável proposta pela Comissão Mundial de Ambiente e Desenvolvimento (Wecd), segundo Véron (2001), traz na primeira parte um contexto relativo ao objetivo de desenvolvimento econômico e social convencional e incorpora na segunda parte a visão de longo prazo, incluindo considerações sobre questões ambientais. No conceito de desenvolvimento sustentável é comum a inclusão de quatro elementos: capital natural, capital físico ou produtivo, capital humano e capital social. A recente inclusão do capital social trouxe as questões da confiança, normas, reciprocidade e redes de engajamento civis como forças para o sucesso do desenvolvimento (Véron, 2001).

Do conceito de desenvolvimento sustentável emergiram as discussões sobre a sustentabilidade, passando esta temática a ser amplamente discutida em vários campos do conhecimento. Do ponto de vista da atividade econômica humana, como pondera Walter (2002), a sustentabilidade pode ser vista como uma meta básica, que é implicitamente assumida no contexto mundial, e, frequentemente, é levada à dimensão nacional ou ao menor nível de detalhe geográfico, trazendo o empoderamento das comunidades-locais e o desenvolvimento das capacidades dos 
indivíduos para lidar com as demandas ambientais.

Walter (2002) enfatiza ainda que os limites para crescimento e exploração econômica, impostos pelos recursos ecológicos de uma comunidade, criam uma necessidade de soluções que envolvem a descoberta e a reconciliação de valores. Isto requer atenção ao processo de compromisso mútuo de agentes econômicos, incluindo os ativos humano, natural e informacional local. Véron (2001) complementa que a sustentabilidade, sob a ótica da dimensão social, inclui o estreitamento das comunidades-base para ação coletiva, com vistas ao desenvolvimento sustentável e à sustentabilidade para as gerações presentes e futuras.

Entretanto, como observam Manzini e Vezzolli (2005), o sistema de produção e de consumo das sociedades industriais contemporâneas está distante dos requisitos da sustentabilidade e isto pode ser visualizado pelo uso insensato dos recursos renováveis e dos não-renováveis, pela emissão de um número crescente de novas substâncias sintéticas e nocivas no meio ambiente e pela crescente distância entre os recursos disponíveis para os países mais ricos e os utilizados pelos países mais pobres. Hawken et al. (1999, p.3) destacam que o "[...]estoque de capital natural vem diminuindo e os serviços fundamentais de geração de vida que dele fluem estão se tornando críticos no que diz respeito a nossa prosperidade".

O relacionamento entre o sistema socioeconômico e o sistema ambiental pode ser representado por uma via de mão-dupla. Na visão Graft et al. (1996) existe um limite de uso de bens e serviços que, se for excedido, pode resultar em consequências catastróficas. Para ele, a via de mão-dupla se expressa pelo fato de que o sistema econômico seria o responsável pela gestão ambiental com menos impacto se retirasse do sistema ambiental uma produção de bens e serviços com menos risco e perigo. É importante mencionar que, para estes mesmos autores, o sistema socioeconômico é visto como um misto de processos e estrutura econômica, social e cultural e o sistema ambiental como um misto de processos físicos e ecológicos.

Seguindo esta lógica, a transição do modelo convencional para o sustentável (Manzini e Vezzoli, 2005) prevê uma descontinuidade sistêmica que deve atingir a dimensão física (fluxos de matéria e energia), mas também a dimensão econômica e institucional (as relações entre ao atores sociais), além da dimensão ética, estética e cultural (os critérios de valor e juízos de qualidade que socialmente legitimam o sistema).

A partir deste novo contexto, as publicações sobre a sustentabilidade permitem ponderar que este é um conceito em construção, que se apodera de várias dimensões, onde se destacam a econômica, a social e a ambiental. Para Gladwin, Kennelly e Krause (1995), os estudos sobre sustentabilidade se encontram num âmbito muito subjetivo, permitindo a propagação de diversas interpretações sobre o assunto. No entanto, estes mesmos autores, identificam alguns componentes similares, que permeiam a maioria das publicações sobre o tema, onde aparecem aspectos relacionados à maximização simultânea dos sistemas biológicos, econômicos e sociais, bem como, o incremento da qualidade de vida humana, numa ótica ecossistêmica suportável. Contudo, cientes de que o debate sobre sustentabilidade é recente e que ainda haverá muitas análises neste contexto, sugerem cinco componentes básicos como diretrizes para futuros estudos, que são: inclusão, conectividade, equidade, prudência e segurança.

As argumentações discutidas nesta breve revisão da literatura permitem depreender que a sustentabilidade é um processo de longo prazo que tem relação com componentes de natureza social, relacional, ambiental, ecológica, ética, cultural, estética, econômica, física, material, entre outras. Além disso, a sustentabilidade tem como foco o ecossistema e a sua manutenção para as gerações presentes e futuras, de modo que os recursos naturais, tais como, solo, água, florestas, clima, entre outros, estão no centro das discussões sobre este tema.

\section{MÉTODO DE PESQUISA}

Este estudo pode ser classificado como exploratório e descritivo (Gil, 1999), com análise predominantemente quantitativa. Os procedimentos de pesquisa estão apresentados na Figura 1, a

Revista de Gestão Social e Ambiental - RGSA, São Paulo, v. 5, n. 2, p. 34-46, mai./ago. 2011. 
seguir, onde é possível visualizar que, na primeira fase, foi feita a primeira busca no sistema Web of Science para identificar os assuntos relacionados com a temática central do estudo. O resultado desta primeira busca permitiu organizar, com a literatura estudada, um conjunto mínimo de tópicos a ser combinado com a palavra sustainability, a fim de localizar as publicações sobre este tema no banco de dados.

Após a delimitação do escopo de pesquisa, como se convencionou denominar este conjunto de tópicos, a segunda fase, contemplou a segunda busca ao sistema, combinando cada um dos tópicos com a palavra sustainability, localizando as publicações no período de 1990 a 2006. De posse das publicações, executou-se a terceira fase, onde foi realizada a classificação das publicações e a identificação dos tópicos quentes. Para a classificação, utilizou-se o dispositivo analyze do banco Web of Science classificando as publicações por: country/territory, institution name, language, publication year, source title, subject category. Este sistema computava os resultados para 100.000 documentos e, neste caso, foram selecionados os 25 resultados mais frequentes.

Para a identificação dos tópicos quentes, utilizou-se o dispositivo sort by/times cited, a fim de identificar os artigos mais citados, e o dispositivo publication years para identificar o ano das publicações e proceder ao cálculo do h-b index e do valor de $\mathrm{m}$, seguindo a metodologia proposta por Banks (2006).

A discussão em torno da relevância da produção científica de um determinado autor, levou Hirsch (2005) a apresentar o índice $h$ que, partindo das publicações mais citadas, avalia a produtividade de um pesquisador e o impacto de seus trabalhos sobre a ciência ao longo de sua carreira. Banks (2006) aproveitou as qualidades do índice h e propôs o h-b index, que é uma derivação do h, para a mensuração de tópicos e compostos, no caso, na área de física. Segundo este mesmo autor, o índice $h-b$ aplica-se na busca de tópicos recentes e de fronteira para a realização de pesquisas, auxiliando estudantes de pós-graduação na escolha de seu objeto de investigação. O h-b é calculado a partir do volume de citações de um tópico no período analisado e corresponde ao número onde o número de citações é maior ou igual ao número de ordem do tópico. Por exemplo, um índice h-b de 15 quer dizer que os 15 artigos mais citados possuem, no mínimo, 15 citações cada. A partir do h-b pode-se fazer a relação com o tempo decorrente desde a primeira publicação sobre aquele tópico, calculando-se assim o valor de $\mathrm{m}(\mathrm{m}=\mathrm{h}-\mathrm{b} / \mathrm{n})$, que permite classificar os tópicos. 


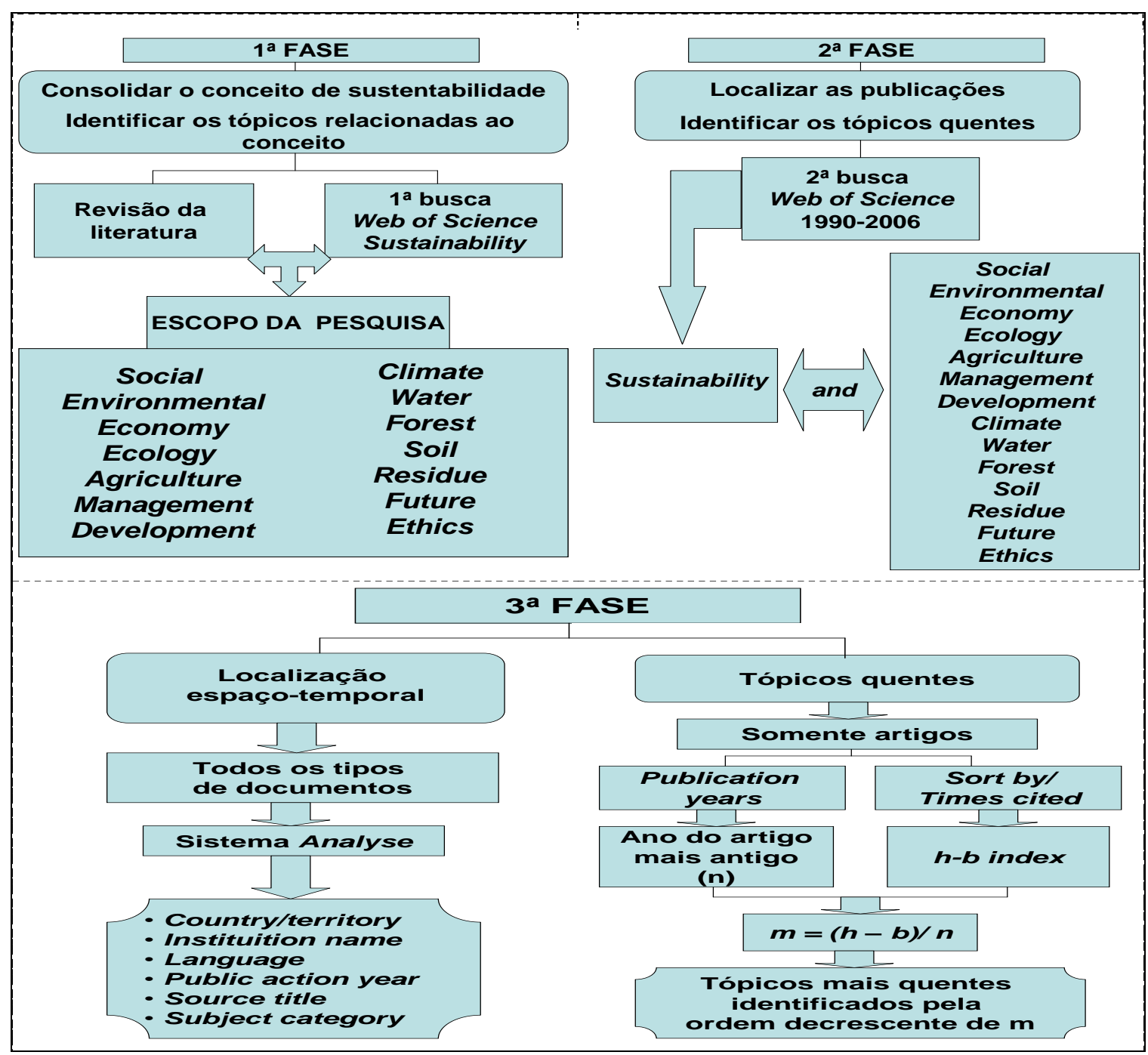

Figura 1: Procedimentos da pesquisa.

Fonte: Elaborado pelos autores.

As palavras utilizadas no Web of Science, assim como os títulos de seus sistemas e dispositivos operacionais, foram mantidos no idioma inglês para facilitar a repetição do procedimento por outras pessoas interessadas.

\subsection{A delimitação do escopo de pesquisa}

O procedimento adotado para definir o escopo da pesquisa merece uma seção especial, por ser considerado um ponto chave no desenvolvimento deste estudo. O caráter multidisciplinar da sustentabilidade, enfatizado no referencial teórico, sinalizou para uma importante relação desta temática com disciplinas, tais como economia, ecologia, agricultura, gestão e desenvolvimento, assim como a temas ligados ao estudo dos ecossistemas, tais como clima, florestas, água, entre outros. Embora muitas outras dimensões tenham sido incorporadas ao conceito de sustentabilidade foram fortemente evidenciadas as dimensões social e ambiental.

Está presente no discurso dos autores estudados que a sustentabilidade é uma questão de longo prazo, que demanda uma nova forma de agir em relação aos recursos naturais, pautada na preservação e conservação do meio ambiente. Isto remete, naturalmente, ao estudo da ética e da sustentabilidade no futuro, assim como à questão dos resíduos decorrentes das atividades industriais e antrópicas.

A primeira busca no Web of Science utilizando-se a palavra sustainability, como evidenciado na Figura 1, permitiu identificar as principais fontes e assuntos ligados a este tema, 
onde as dez fontes e assuntos mais frequentes estão apresentados, em ordem de importância, na Figura 2.

\begin{tabular}{|l|l|}
\hline \multicolumn{1}{|c|}{ FONTE } & ASSUNTO \\
\hline Ecological economics & Environmental sciences \\
\hline $\begin{array}{l}\text { Agriculture ecosystems \& } \\
\text { environment }\end{array}$ & Ecology \\
\hline Water science and technology & Economics \\
\hline Forest ecology and management & Environmental studies \\
\hline $\begin{array}{l}\text { International journal of sustainable } \\
\text { development and world ecology }\end{array}$ & Agriculture, multidisciplinary \\
\hline Journal of sustainable agriculture & Water resources \\
\hline Environmental management & Planning and development \\
\hline Forestry chronicle & Engineering, environmental \\
\hline Ambio & Forestry \\
\hline World development & Agronomy \\
\hline
\end{tabular}

Figura 2: Principais fontes e assuntos evidenciados no Web of Science usando o descritor sustainability.

Fonte: Web of Science, acesso em 05/06/2006.

Como demonstrado na Figura 2, as publicações sobre sustentabilidade, disponíveis Web of Science, se relacionam a temas, tais como ecologia, agricultura, ambiente, florestas, gestão, desenvolvimento, economia, água, entre outros, conforme evidenciado pelos títulos das principais fontes e pelos principais assuntos, categorizados por este banco de dados.

Diante do universo de possibilidades evidenciado, tanto na literatura quanto nas publicações do Web of Science, considerou-se pertinente iniciar o estudo pelo escopo de pesquisa demonstrado na Figura 3, sem a pretensão de esgotar o assunto, mas sim de apresentar uma primeira tentativa para delinear uma metodologia que possa ser utilizada por outros pesquisadores no estudo de temáticas pertinentes à sua área de interesse. Para tanto, os tópicos foram categorizados em: disciplinas, dimensões conceituais, elementos do ecossistema, resultados e temas transversais, a critério do pesquisador.

Outro aspecto que merece destaque é o fato de que o critério de escolha dos tópicos a serem relacionados com a sustentabilidade foi subjetivo e baseado na percepção do pesquisador, reforçado pela construção teórica e pelas informações do banco de dados e também pelo contexto das disciplinas de Gestão Ambiental (PPGA/UFRGS), Economia Ecológica (PGDR/UFGRS) e Tópicos Avançados I (PPG Agronegócios/UFRGS), cursadas no primeiro semestre de 2006, onde os professores podem ser considerados especialistas e cujas opiniões foram utilizadas indiretamente neste trabalho. 


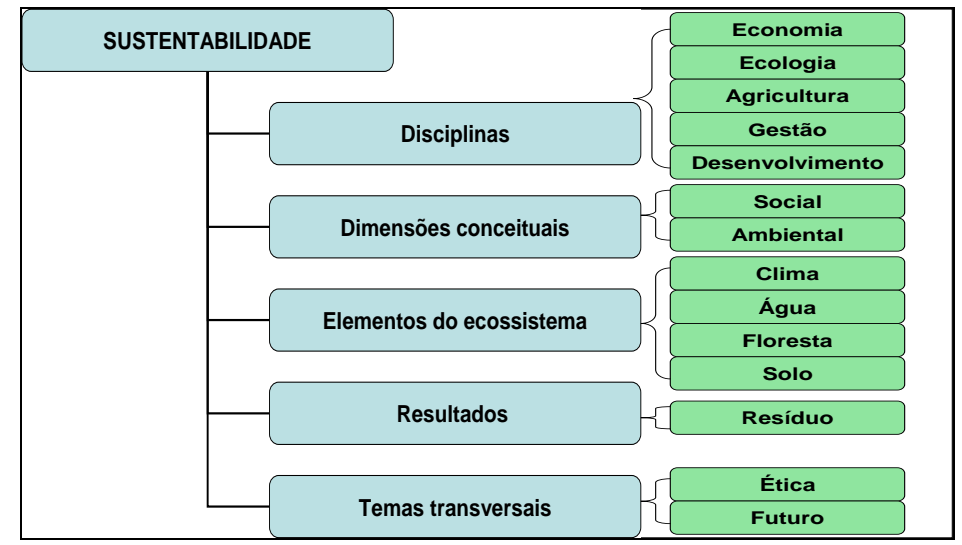

Figura 3: Escopo de pesquisa.

Fonte: Elaborado pelos autores

O critério de escolha, entretanto, pode ser refinado pelo uso de outros sistemas da base de dados do Portal da Capes, como o banco de dados Scopus, que apresenta palavras-chave relacionadas com qualquer tema a ser investigado. A divisão dos tópicos em categorias é um recurso didático empregado com a finalidade de reunir os elementos com fins e/ou características de relacionamentos semelhantes com a temática da sustentabilidade.

\section{SUSTENTABILIDADE: A LOCALIZAÇÃO ESPAÇO-TEMPORAL DAS PUBLICAÇÕES}

Nesta seção, apresentam-se os resultados da pesquisa com destaque para a localização das publicações e para a identificação dos tópicos quentes na área da sustentabilidade.

\subsection{Onde se localizam as publicações}

A Figura 4 apresenta um quadro resumo com o número de publicações encontradas em cada combinação, considerando-se qualquer tipo de documento, entre 1990 e 2006.

\begin{tabular}{|l|r|}
\hline \multicolumn{1}{|c|}{ Busca no sistema } & № de publicações \\
\hline TS=(sustainability) & 10.932 \\
\hline TS=(sustainability and economy) & 606 \\
\hline TS=(sustainability and ecology) & 545 \\
\hline TS=(sustainability and agriculture) & 1.176 \\
\hline TS=(sustainability and management) & 3.684 \\
\hline TS=(sustainability and development) & 3.421 \\
\hline TS=(sustainability and social) & 1.747 \\
\hline TS=(sustainability and environmental) & 3.223 \\
\hline TS=(sustainability and climate) & 1.612 \\
\hline TS=(sustainability and water) & 998 \\
\hline TS=(sustainability and forest) & 1.557 \\
\hline TS=(sustainability and soil) & 125 \\
\hline TS=(sustainability and residue) & 1.589 \\
\hline TS=(sustainability and future) & 159 \\
\hline TS=(sustainability and ethics)
\end{tabular}

Figura 4: Número de publicações para cada combinação.

Fonte: Web of Science acesso no período de 22/05/06 a 05/06/06 para DocType=All document types; Language =All languages; Databases $=$ SCI-EXPANDED, SSCI, A\&HCI; Timespan=1990-

Revista de Gestão Social e Ambiental - RGSA, São Paulo, v. 5, n. 2, p. 34-46, mai./ago. 2011. 


\section{6}

A Figura 4 evidencia que o maior volume de publicações sobre sustentabilidade, no sistema Web of Science, é encontrado quando se relaciona este tema com a palavra management, totalizando 3.684 publicações, entre 1990 e 2006. O segundo maior volume de publicações refere-se à palavra development, com 3.421 publicações no mesmo período, ambas categorizadas, neste estudo, como disciplinas; e o terceiro maior volume está relacionado à palavra environmental, categorizada como dimensão conceitual.

\subsection{Análise por país e idioma}

A análise por país e idioma foi organizada buscando-se os países e os idiomas mais frequentes no primeiro, segundo, terceiro e quarto lugares, conforme a classificação do sistema analyze. Neste caso, ficou evidente a supremacia dos Estados Unidos (EUA) sobre os demais países. Em todos os relacionamentos este país ficou em primeiro lugar com percentuais bastante expressivos, se comparados com os dos demais países. Em segundo lugar ficou a Inglaterra; em terceiro, o Canadá e; em quarto lugar, a Austrália, com percentuais de menor expressividade.

$\mathrm{O}$ idioma predominante em todos os relacionamentos foi o inglês, com percentuais expressivamente maiores que a segunda língua mais frequente que foi a língua alemã. Em terceiro lugar, com pouca expressividade em termos percentuais, apareceu o espanhol. O idioma português foi citado em segundo lugar, duas vezes e, em terceiro lugar, três vezes. As citações em segundo lugar aconteceram para os relacionamentos de sustentabilidade com água e solo e, as citações em terceiro lugar foram relacionadas à floresta, clima e ecologia.

É importante mencionar que o Brasil apareceu em $25^{\circ}$ lugar no relacionamento entre sustentabilidade e ecologia com 4 publicações e, em $11^{\circ}$, no relacionamento entre sustentabilidade e solo, com 58 publicações.

\subsection{Análise por ano}

A Figura 5 apresenta as publicações sobre a sustentabilidade, no período de 1990 a 2006, usando-se o descritor sustainability, e demonstra que houve um crescimento do percentual de publicações nos anos mais recentes.

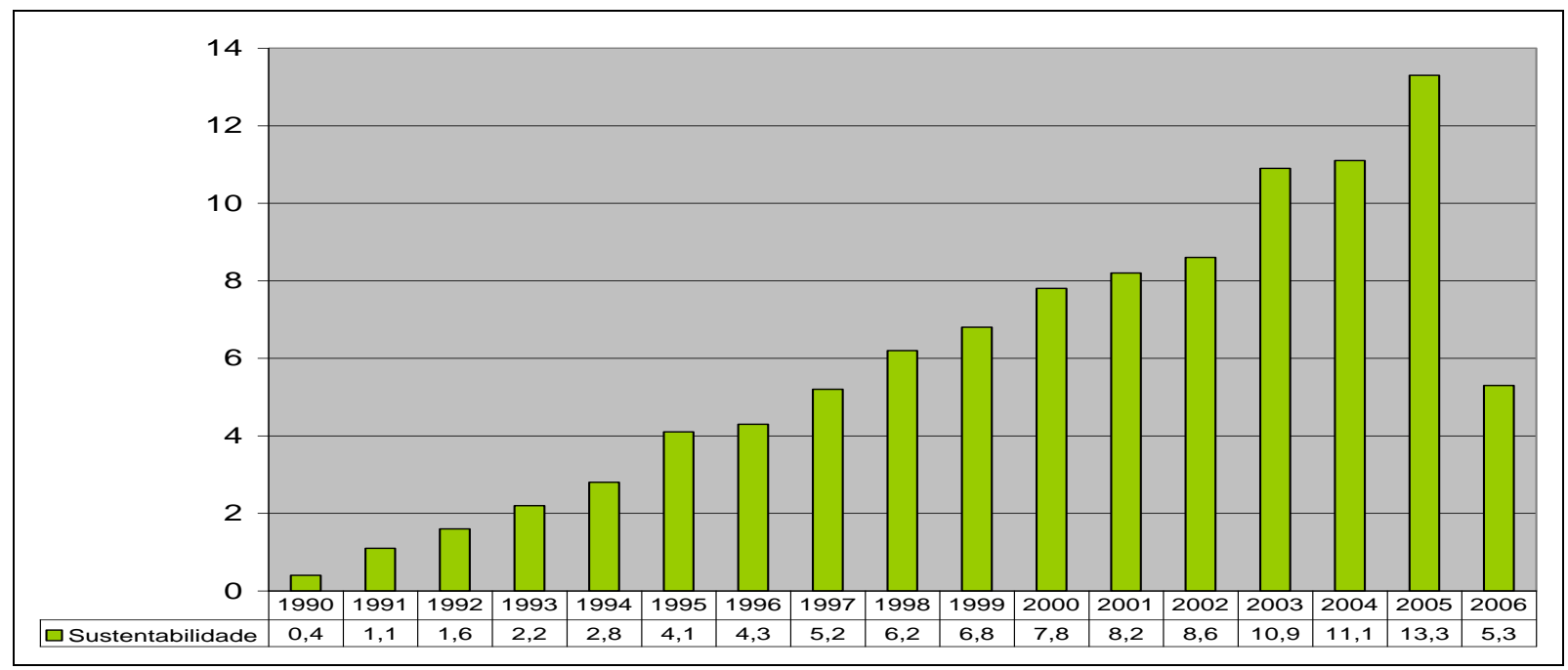

Figura 5: Publicações sobre sustentabilidade usando o descritor sustainability.

Fonte: Web of Science acesso no período de 22/05/06 a 05/06/06.

A Figura 5 permite visualizar que o percentual de publicações por ano, praticamente dobrou, de 1997 para 2005 e, se for considerado o percentual de publicações no início dos anos 1990, comparativamente à década de 2000 , verifica-se um aumento de mais de quatro vezes. Merece 
destaque o fato de que, em 2006, já aparecem 1.737 publicações incluindo todos os temas. Na Figura 6, é possível verificar que a tendência de crescimento, observada quando se busca somente o termo sustainability e quando se combina este termo com os tópicos do escopo de pesquisa, é mantida em todas as combinações. Merece atenção a ética, que duplicou o número de publicações de 2004 para 2005.

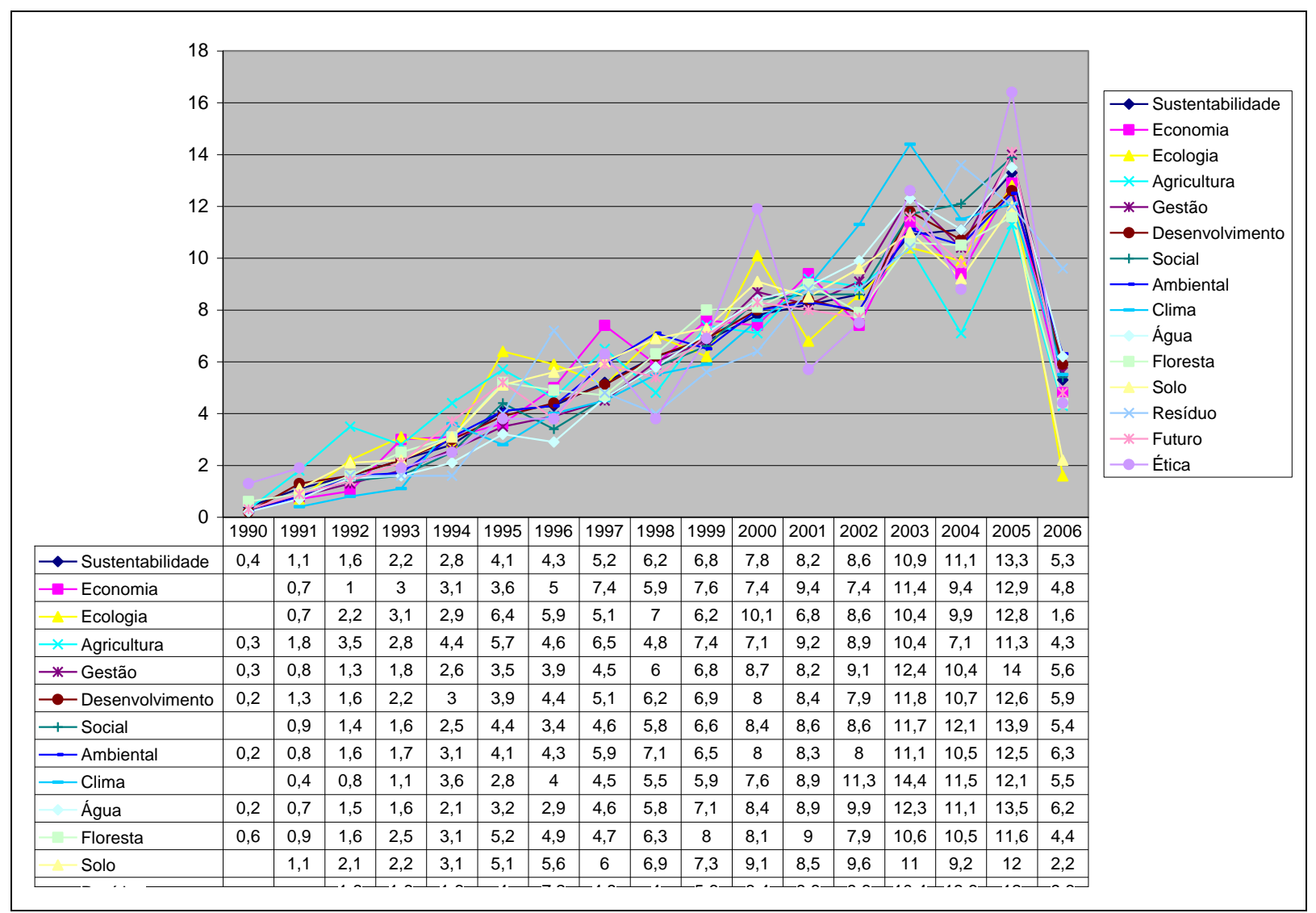

Figura 6: Publicações sobre sustentabilidade combinando o descritor sustainability com cada tópico do escopo de pesquisa.

Fonte: Web of Science acesso no período de 22/05/06 a 05/06/06.

Para verificar quais os temas mais correlacionados com o crescimento das publicações sobre sustentabilidade, procedeu-se a uma análise de correlação entre o número de publicações sobre sustentabilidade por ano e o número de publicações em cada um dos temas. Todos os temas apresentaram uma forte correlação positiva com a temática da sustentabilidade, sendo a maior correlação registrada com a dimensão conceitual relativa ao social $(0,995)$, seguida das disciplinas de desenvolvimento $(0,994)$ e de administração/gestão $(0,992)$. Tal resultado está ilustrado graficamente na Figura 7 e pode evidenciar a crescente preocupação da sociedade e da comunidade cientifica em incluir as questões sociais como importantes indicadores para a prática da sustentabilidade, reforçando o papel dos agentes sociais.

No caso dos elementos do ecossistema, a maior correlação foi com a água $(0,988)$ e, nos temas transversais, com as questões de futuro $(0,983)$. 


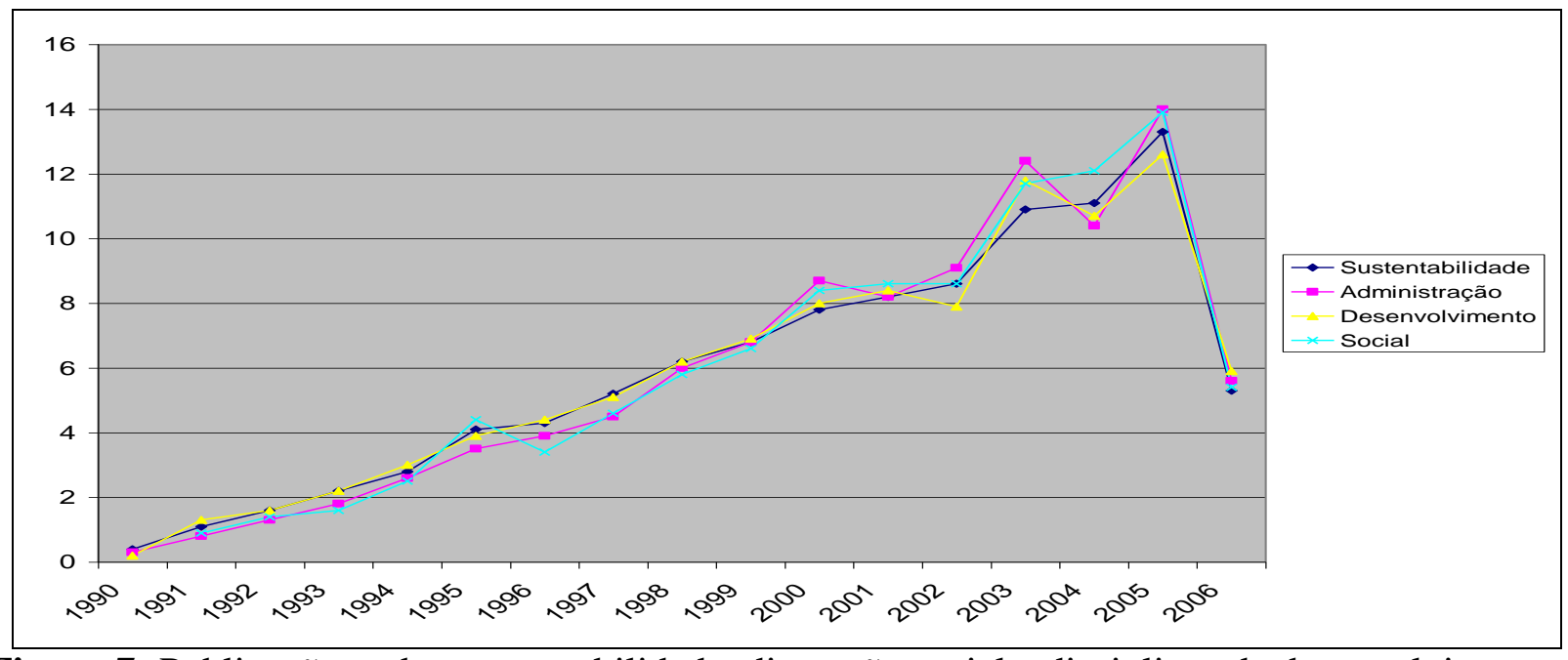

Figura 7: Publicações sobre sustentabilidade, dimensão social e disciplinas de desenvolvimento e gestão.

Fonte: Web of Science acesso no período de 22/05/06 a 05/06/06.

As menores correlações, embora bastante expressivas, aconteceram nas questões dos resíduos $(0,881)$ e da ética $(0,904)$.

\subsection{Análise por instituição, fonte e assunto}

As análises por instituição, fonte e assunto privilegiaram o primeiro resultado mais importante, evidenciado pelas estatísticas do banco de dados.

\begin{tabular}{|c|c|c|c|}
\hline Combinação & Instituição & Fonte & Assunto \\
\hline Sustentabilidade & Univ British Columbia & Ecological Economics & Environmental Sciences \\
\hline \multicolumn{4}{|l|}{ Disciplinas } \\
\hline Economia & Free Univ Amsterdam & Ecological Economics & Environmental Sciences \\
\hline Ecologia & Univ British Columbia & Landscape and Urban Planning & Ecology \\
\hline Agricultura & Cornell Univ & Agriculture Ecosystems \& Environment & Environmental Sciences \\
\hline Administração & Usda Ars & Forest Ecology and Management & Environmental Sciences \\
\hline Desenvolvimento & Univ British Columbia & Ecological Economics & Environmental Sciences \\
\hline \multicolumn{4}{|c|}{ Dimensões Conceituais } \\
\hline Social & Univ British Columbia & Ecological Economics & Environmental Sciences \\
\hline Ambiental & Univ British Columbia & Ecological Economics & Environmental Sciences \\
\hline \multicolumn{4}{|c|}{ Elementos Do Ecossistema } \\
\hline Clima & Univ British Columbia & Agriculture Ecosystems \& Environment & Environmental Sciences \\
\hline Água & Usda Ars & Water Science and Technology & Environmental Sciences \\
\hline Floresta & Us Forest Serv & Forest Ecology and Management & Forestry \\
\hline Solo & Usda Ars & Agriculture Ecosystems \& Environment & Agriculture, Soil Science \\
\hline \multicolumn{4}{|l|}{ Resultados } \\
\hline Resíduo & Usda Ars & Soil \& Tillage Research & Agriculture, Soil Science \\
\hline \multicolumn{4}{|c|}{ Temas Transversais } \\
\hline Futuro & Univ British Columbia & Ecological Economics & Environmental Sciences \\
\hline Ética & Univ British Columbia & $\begin{array}{l}\text { Journal Of Agricultural \& } \\
\text { Environmental Ethics }\end{array}$ & Environmental Sciences \\
\hline
\end{tabular}

Figura 8: Instituições, fontes e assuntos mais importantes.

Fonte: Web of Science acesso no período de 22/05/06 a 05/06/06.

Revista de Gestão Social e Ambiental - RGSA, São Paulo, v. 5, n. 2, p. 34-46, mai./ago. 2011. 
A Figura 8 resume os resultados obtidos para cada combinação e demonstra que uma instituição bastante forte na pesquisa sobre sustentabilidade é a University British Columbia, que aparece em primeiro lugar em $47 \%$ das combinações. Quanto à fonte, destaca-se o Journal Ecological Economics, que aparece em primeiro lugar em $40 \%$ das combinações.

Quanto ao assunto, verifica-se, na Figura 8, que grande parte das publicações tem relação com as ciências ambientais, que aparecerem em primeiro lugar em onze das combinações realizadas (73\%). É relevante o fato de que, nas publicações sobre a dimensão social e econômica, as ciências ambientais surgem como o primeiro assunto, denotando que o apelo ambiental pode estar sensibilizando outras áreas do conhecimento.

As análises por instituição, fonte e assunto reforçaram a importância do contexto ambiental e da visão multidisciplinar da temática central. Percebe-se que os estudos sobre resíduos estão publicados em fontes ligadas aos estudos do solo. Nos estudos de futuro aparecem questões relativas à água e às florestas. Nos estudos sobre florestas destacam-se os aspectos de gestão, solo e ecossistemas. Nos estudos sobre gestão surgem os aspectos relacionados às florestas, à água e à agricultura.

\subsection{Identificação dos tópicos quentes}

A identificação dos tópicos quentes foi feita utilizando-se o sistema Web of Science e seguiu a metodologia do h-b index, proposta por Banks (2006). Na Figura 9, é possível visualizar que, para o escopo de pesquisa analisado, os tópicos mais quentes referem-se às disciplinas de gestão $(2,625)$ e desenvolvimento (2,500); à dimensão ambiental (2,375) e aos elementos do ecossistema, solo $(2,312)$ e água $(2,188)$.

\begin{tabular}{|l|r|r|r|c|}
\hline Tema & $\begin{array}{c}\text { Número de } \\
\text { artigos }\end{array}$ & $\boldsymbol{h}$ - $\boldsymbol{~}$ & Número anos (n) & $\mathbf{m}=\mathbf{h}-\mathbf{b} / \mathbf{n}$ \\
\hline Management & $\mathbf{3 . 3 6 4}$ & $\mathbf{4 2}$ & $\mathbf{1 6}$ & $\mathbf{2 , 6 2 5}$ \\
\hline Development & $\mathbf{3 . 1 0 9}$ & $\mathbf{4 0}$ & $\mathbf{1 6}$ & $\mathbf{2 , 5 0 0}$ \\
\hline Environmental & $\mathbf{2 . 8 7 6}$ & $\mathbf{3 8}$ & $\mathbf{1 6}$ & $\mathbf{2 , 3 7 5}$ \\
\hline Soil & $\mathbf{1 . 4 9 1}$ & $\mathbf{3 7}$ & $\mathbf{1 6}$ & $\mathbf{2 , 3 1 2}$ \\
\hline Water & $\mathbf{1 . 0 4 7}$ & $\mathbf{3 5}$ & $\mathbf{1 6}$ & $\mathbf{2 , 1 8 8}$ \\
\hline Agriculture & 1.046 & 31 & 16 & 1,938 \\
\hline Forest & 919 & 31 & 16 & 1,938 \\
\hline Future & 1.435 & 31 & 16 & 1,938 \\
\hline Social & 1.612 & 30 & 16 & 1,875 \\
\hline Ecology & 461 & 26 & 15 & 1,733 \\
\hline Economy & 563 & 22 & 15 & 1,470 \\
\hline Residue & 126 & 16 & 12 & 1,333 \\
\hline Climate & 460 & 21 & 16 & 1,312 \\
\hline Ethics & 135 & 12 & 13 & 0,920 \\
\hline
\end{tabular}

Figura 9: Tópicos quentes em sustentabilidade.

Fonte: Web of Science acesso em 30/06/06 restrito a artigos.

O resultado da pesquisa relativa aos tópicos quentes reflete as constatações já evidenciadas anteriormente. $\mathrm{Na}$ análise por ano, as disciplinas de gestão e desenvolvimento se destacaram como as mais correlacionadas com o crescimento das publicações sobre sustentabilidade, assim como a água, no caso dos elementos do ecossistema. Na análise por assunto, a dimensão ambiental já evidenciava as ciências ambientais como o assunto predominante, que apareceu em primeiro lugar em $73 \%$ das combinações. 


\section{CONSIDERAÇÕES FINAIS}

Esse estudo revelou o caráter multidisciplinar da sustentabilidade, indicando que trata-se de um conceito em construção que abarca muitas outras temáticas, especialmente aquelas que se relacionam com as questões ambientais e a dimensão social. Além disso, demonstrou que nos últimos anos este tema apresentou um volume crescente de publicações.

Os dados revelaram que as publicações concentram-se, prioritariamente, nos Estados Unidos, com a predominância do idioma inglês, sendo que, entre as principais instituições, destacase a University British Columbia.

Como principal fonte destacou-se o Journal Ecological Economics e, como principal assunto, as ciências ambientais, que são o assunto predominante em áreas como a econômica e a social. Na identificação dos tópicos quentes pode-se dizer que os temas considerados de fronteira são relacionados às disciplinas de gestão e desenvolvimento, à dimensão ambiental e, à água e ao solo. Neste caso, também se depreendeu que a gestão florestal é um tema relevante.

Esse estudo contribui com outras temáticas na pesquisa multidisciplinar, na medida em que se procurou desenvolver um conjunto de procedimentos que se iniciam com a discussão sobre o escopo de pesquisa a ser utilizado para chegar ao conjunto das publicações na área de interesse do pesquisador, seguindo com uma proposição de como conduzir uma análise espaço-temporal das publicações.

É importante destacar que este trabalho não pode ser dado como finalizado, pois existem várias formas de aprofundá-lo. Com a mesma base de dados, é possível, entre outras maneiras, continuá-lo pela ampliação do escopo de pesquisa. Ainda é possível aprofundar este estudo, partindo dos tópicos quentes e focalizando as principais temáticas em cada um deles, permitindo a identificação de suas principais decorrências e implicações. Outra forma de continuidade pode ser pela utilização de outra base de dados a fim de complementar, comparar ou substituir os resultados obtidos neste trabalho.

\section{REFERÊNCIAS}

Banks, M.G. [2006]."An Extension of the hirsch index: Indexing scientific topics and compounds". Recuperado em 2 junho 2006, de: www.arxiv.org/abs/physics/0604216.

Gil, A. C. (1999) Métodos e técnicas de pesquisa social. (5 ed.) São Paulo: Atlas.

Gladwin, T., Kennelly, J. J. \& Krause, T. (1995). Shifting paradigms for sustainable development: implications for management theory and research. Academy of Management Review. 20 (4): 874907.

Graft, H. J., Musters, C. J. M \& Keurs, W. J. (1996). Sustainable development: looking for new strategies. Ecological Economics, 16, 205-216.

Hawken, P., Lovins, A., Lovins, L. H. (1999) Capitalismo natural: criando a próxima revolução industrial. São Paulo: Cultrix.

Hirsch, J. E. (2005). An index to quantify an individual's scientific research output. Proc. Nat. Acad. Sci. 102, 165- 169.

Manzini, E., Vezzoli, C.(2005) O desenvolvimento de produtos sustentáveis. Tradução de Astrid Carvalho. São Paulo: Editora da Universidade de São Paulo. 
Thompson/Institute for Scientific Information. Base Referencial Web of Science. Philadelphia. Recuperado em maio/junho 2006, de: http://portal.isiknowledge.com/portal.cgi?DestApp=WOS\&Func=Frame .

Véron, R. (2001) “The 'new' Kerala model: lessons for sustainable development". World Development. 29 (4), 601-617.

Vieira, V.A. (2002). As tipologias, variações e características da pesquisa de marketing. Revista FAE, Curitiba. 5 (1): $61-70$.

Walter, G.R. (2002) Economics, ecology-based communities, and sustainability. Ecological Economics. 42: 81-87.

Data do recebimento do artigo: 03/06/2011

Data do aceite de publicação: 08/08/2011 